\title{
A method for augmenting supersaturated designs
}

\author{
Qiao-Zhen Zhang ${ }^{1}$, Hong-Sheng Dai ${ }^{2}$, Min-Qian Liu ${ }^{1 *}$ Ya Wang ${ }^{3}$ \\ ${ }^{1}$ LPMC and Institute of Statistics, Nankai University, Tianjin 300071, China \\ ${ }^{2}$ Department of Mathematical Sciences, University of Essex, Wivenhoe Park, \\ Colchester, $\mathrm{CO}_{4} 3 \mathrm{SQ}$, UK \\ ${ }^{3}$ State Key Laboratory of Complex Electromagnetic Environment Effects on \\ Electronics and Information System (CEMEE), Luoyang, 471003, China
}

\begin{abstract}
Initial screening experiments often leave some problems unresolved, adding follow-up runs is needed to clarify the initial results. In this paper, a technique is developed to add additional experimental runs to an initial supersaturated design. The added runs are generated with respect to the Bayesian $D_{s}$-optimality criterion and the procedure can incorporate the model information from the initial design. After analysis of the initial experiment with several methods, factors are classified into three groups: primary, secondary, and potential according to the times that they have been identified. The focus is on those secondary factors since they have been identified several times but not so many that experimenters are sure that they are active, the proposed Bayesian $D_{s}$-optimal augmented design would minimize the error variances of the parameter estimators of secondary factors. In addition, a blocking factor will be involved to describe the mean shift between two stages. Simulation results show that the method performs very well in certain settings.
\end{abstract}

Keywords: Bayesian D-optimality; Coordinate-exchange algorithm; Follow-up experiment; Sequential design; Supersaturated design.

\footnotetext{
*Corresponding author. Email address: mqliu@nankai.edu.cn (Min-Qian Liu).
} 


\section{Introduction}

Screening is the first phase of an experimental study on systems and simulation models. Its purpose is to eliminate negligible factors so that efforts may be concentrated upon just the important ones (active factors). Using a supersaturated design (SSD) whose run size is not enough for estimating all the main effects may be considered when a large experiment is infeasible in practice. SSDs were introduced by Box (1959), but not studied further until the appearance of the work by $\operatorname{Lin}(1993)$ and $\mathrm{Wu}(1993)$. Many developments in the area have taken place over the last two decades. For further details, please refer to Georgiou (2014), Sun et al. (2011) and the references therein.

The analysis of SSDs is challenging due to the inherent non-full rank nature of the design matrix and the fact that the columns of the model matrix are correlated. As a result, the effects of different factors are aliased with one another making it very difficult to identify the active factors correctly. Methods to overcome these problems include regression procedures, such as forward selection (Westfall et al. 1998), stepwise and all-subsets regression (Abraham et al. 1999), partial least squares methods (Zhang et al., 2007; Yin et al., 2013), shrinkage methods, including SCAD (Li and Lin, 2002) and Dantzig selector (Phoa et al., 2009) and Bayesian methods (Beattie et al., 2002; Chen et al., 2011, 2013; Huang et al., 2014). Readers can refer to Salawu et al. (2015) and Georgiou (2014). However, different methods may give different results and no method is infallible.

If we want to clarify or confirm initial results and guide the next phase of experimentation, adding follow-up runs to the initial design is a useful way. As a matter of fact, performing extra experimental runs is the only data-driven way to break confounding patterns and to disentangle confounded effects. Suppose an SSD of $n_{1}$ runs and $k$ 2-level factors, denoted by $\operatorname{SSD}\left(n_{1}, k\right)$, has been run and now the experimenter can afford $n_{2}$ more runs to resolve ambiguities, the target is to find the best way to augment the original design to reduce uncertainty and get the most information out of the final $\operatorname{SSD}\left(n_{1}+n_{2}, k\right)$. Gupta et al. (2010) considered the problem for 2-level SSDs firstly: $E\left(s^{2}\right)$-optimal designs (proposed by Booth and Cox, 1962) are augmented with additional runs to create a new class of "extended 
$E\left(s^{2}\right)$-optimal" designs. Then Gupta et al. (2012) extended the method to $s$-level designs. Suen and Das (2010) also used a similar approach to add or remove one row from an existing $E\left(s^{2}\right)$-optimal design to make a new $E\left(s^{2}\right)$-optimal design. Qin et al. (2015) studied the optimality of the extended design generated by adding few runs to an existing $E\left(\chi^{2}\right)$-optimal mixed-level SSD and their paper covers the work of Gupta et al. $(2010,2012)$ as two special cases. All of these methods, however, did not consider using the information from the initial analysis and design when adding runs. Gutman et al. (2014) proposed an SSD augmentation strategy using the Bayesian $D$-optimality criterion, they considered the information gained from the initial design, $\operatorname{SSD}\left(n_{1}, k\right)$, as a prior, and constructed the final $\operatorname{SSD}\left(n_{1}+n_{2}, k\right)$ to reduce the error variances of the parameter estimators under the Bayesian paradigm.

When adding runs to fractional factorial designs, two optimality criteria, $D$-optimality and $D_{s^{-}}$-optimality, are often used. The $D_{s}$-optimal design approach would be applied if the experimenters emphasize precise estimation for the "subset" of the experimental factors. Kiefer and Wolfowitz (1961) defined a design as $D_{s}$-optimal if it minimizes the determinant of the normalized covariance sub-matrix of estimators of the chosen model parameters while treating the other parameters as nuisance parameters. The use of $D_{s}$-optimality designs would result in increased power since the parameters of interest are estimated more precisely (Atkinson and Donev, 1992; Casey et al., 2005). In this paper, we will combine the $D_{s^{-}}$ optimality criterion with the Bayesian technique to propose an alternative approach, which is different from the Bayesian $D$-optimal augmentation in two aspects: the principle of factor classification and the optimal criterion.

The next section reviews the relevant background firstly, then we propose the new algorithmic augmentation strategy for SSDs using information from the initial runs in Section 3. Section 4 compares the performance of the Bayesian $D_{s}$-optimal augmented designs with the Bayesian $D$-optimal augmented designs by several highlighting examples. Some concluding remarks are provided in Section 5 . 


\section{Bayesian $D$-optimality and model selection methods}

In this section, we briefly review the approach for developing augmenting Bayesian $D$ optimal designs in the context of linear models and some model selection methods applied to SSDs.

\subsection{Bayesian D-optimality}

Consider the linear model

$$
\boldsymbol{y}=\beta_{0} \mathbf{1}_{n}+\beta_{1} \boldsymbol{x}_{1}+\cdots+\beta_{k} \boldsymbol{x}_{k}+\boldsymbol{\varepsilon}=\boldsymbol{X} \boldsymbol{\beta}+\boldsymbol{\varepsilon},
$$

where $\boldsymbol{y}$ is an $n \times 1$ vector of observations, $\beta_{0}$ is the intercept term, $\mathbf{1}_{n}$ is an $n \times 1$ column vector with all elements unity, $\boldsymbol{x}_{i}$ is an $n \times 1$ vector of settings for the $i$ th factor, $\boldsymbol{X}$ is the $n \times p$ design matrix with $p=k+1, \boldsymbol{\beta}$ is the $p \times 1$ vector of coefficients to be estimated, and $\boldsymbol{\varepsilon} \sim N\left(\mathbf{0}_{n}, \sigma^{2} \boldsymbol{I}_{n}\right)$ is the noise vector, where $\mathbf{0}_{n}$ is an $n \times 1$ column vector with all elements zero, and $\boldsymbol{I}_{n}$ is an identity matrix of order $n$. In a two-level factorial design, each factor setting can be coded as \pm 1 (or simply \pm ). Let the prior distribution of the parameters be $\boldsymbol{\beta} \mid \sigma^{2} \sim N\left(\boldsymbol{\beta}_{0}, \sigma^{2} \boldsymbol{R}^{-1}\right.$ ),

where $\boldsymbol{\beta}_{0}$ is the mean of prior distribution for $\boldsymbol{\beta}, \boldsymbol{R}$ is a prior covariance matrix, and the conditional distribution of $\boldsymbol{y}$ given $\boldsymbol{\beta}$ be $\boldsymbol{y} \mid\left(\boldsymbol{\beta}, \sigma^{2}\right) \sim N\left(\boldsymbol{X} \boldsymbol{\beta}, \sigma^{2} \boldsymbol{I}_{n}\right)$. Then the posterior distribution for $\boldsymbol{\beta}$ given $\boldsymbol{y}$ is

$$
\boldsymbol{\beta} \mid \boldsymbol{y} \sim N\left(\boldsymbol{b}, \sigma^{2}\left(\boldsymbol{X}^{T} \boldsymbol{X}+\boldsymbol{R}\right)^{-1}\right),
$$

where $\boldsymbol{b}=\left(\boldsymbol{X}^{T} \boldsymbol{X}+\boldsymbol{R}\right)^{-1}\left(\boldsymbol{X}^{T} \boldsymbol{y}+\boldsymbol{R} \boldsymbol{\beta}_{0}\right)$.

Let $\boldsymbol{X}_{1}$ be a model matrix corresponding to the initial $n_{1}$ runs of an experiment with response vector $\boldsymbol{y}_{1}$, and $\boldsymbol{X}_{2}$ be the additional $n_{2}$ rows with response vector $\boldsymbol{y}_{2}$. That is

$$
\boldsymbol{X}=\left(\begin{array}{c}
\boldsymbol{X}_{1} \\
\boldsymbol{X}_{2}
\end{array}\right), \boldsymbol{y}=\left(\begin{array}{l}
\boldsymbol{y}_{1} \\
\boldsymbol{y}_{2}
\end{array}\right)
$$


Once the data from the first stage have been collected, many different analysis methods can be employed to identify active factors and the information from the analysis may be used as a prior. Gutman et al. (2014) pointed out that the experimenter can classify a factor as primary term (highlighted by an analysis method or many methods), secondary term (if there is an indication the factor may be active, but it is not a predominant), or potential term ( with little evidence to suggest it is active). Then prior distributions would be assigned as follows. Since the primary terms are likely to be active, their coefficients are specified to have a diffuse prior variance tending to infinity (DuMouchel and Jones, 1994), which implies that the primary terms are likely to be much different from zero. On the other hand, potential terms are unlikely to have large effects, and it is proper to assume that they have a relative small variance. For secondary terms, they may or may not be active, so their prior variances should be finite, but larger than that for potential terms. We assume that the factors in $\boldsymbol{X}$ have been reordered after the initial analysis: the intercept and the first $p_{1}-1$ factors are primary terms, the second $p_{2}$ factors are potential terms, and the last $p_{3}$ factors are secondary terms, where $p_{1}+p_{2}+p_{3}=p$. Thus the prior covariance matrix $\boldsymbol{R}$ would be

$$
\boldsymbol{R}=\left(\begin{array}{ccc}
\boldsymbol{0}_{p_{1} \times p_{1}} & \boldsymbol{0}_{p_{1} \times p_{2}} & \boldsymbol{0}_{p_{1} \times p_{3}} \\
\boldsymbol{0}_{p_{2} \times p_{1}} & \boldsymbol{I}_{p_{2}} / \tau^{2} & \boldsymbol{0}_{p_{2} \times p_{3}} \\
\boldsymbol{0}_{p_{3} \times p_{1}} & \boldsymbol{0}_{p_{3} \times p_{2}} & \boldsymbol{I}_{p_{3}} / \gamma^{2}
\end{array}\right)
$$

where $\mathbf{0}_{s \times t}$ is an $s \times t$ matrix with all elements zero, and $\tau<\gamma$ means the secondary terms are more likely to be active than those potential terms. Using the prior information, the augmented design $\operatorname{SSD}\left(n_{1}+n_{2}, k\right)$ is constructed to reduce the error variances of the parameter estimators under the above Bayesian assumptions for the linear model, that is $\boldsymbol{X}_{2}$ is chosen to maximize $\left|\boldsymbol{X}_{1}^{T} \boldsymbol{X}_{1}+\boldsymbol{X}_{2}^{T} \boldsymbol{X}_{2}+\boldsymbol{R}\right|$ to get a Bayesian $D$-optimal augmented SSD.

\subsection{Model selection methods}

We consider four analysis methods here: the half normal plot (Daniel, 1959), the least absolute shrinkage and selection operator (LASSO) proposed by Tibshirani (1996), the s- 
moothly clipped absolute deviation (SCAD) by Fan and Li (2001), and the Dantzig selector (DS) by Candes and Tao (2007).

The half normal plot visually screens factors whose effects seem larger than a random noise. However, the method may fail to indicate any factor as significantly greater than the experimental noise (Gupta et al., 2010), so here we list top $\lfloor k / 2\rfloor$ largest effects which are more likely active than the others, here $\lfloor\cdot\rfloor$ is the rounding down operation.

The LASSO and SCAD are both members of the penalized least squares. A form of penalized least squares is defined as

$$
(\boldsymbol{y}-\boldsymbol{X} \boldsymbol{\beta})^{T}(\boldsymbol{y}-\boldsymbol{X} \boldsymbol{\beta})+\sum_{j=0}^{k} p_{\lambda}\left(\left|\beta_{j}\right|\right)
$$

where $p_{\lambda}(\cdot)$ is a penalty function, and $\lambda$ is a tuning parameter, which can be chosen by a data-driven approach, such as cross-validation (CV) and generalized cross-validation (GCV). The " $l_{1}$ penalty" $p_{\lambda}(|\beta|)=\lambda|\beta|$ results in LASSO. Fan and Li (2001) pointed out that most penalty functions do not simultaneously satisfy the mathematical conditions for unbiasedness, sparsity, and continuity and they suggested using the SCAD penalty proposed by Fan (1997). The first-order derivative of SCAD is defined by

$$
p_{\lambda}^{\prime}(|\beta|)=\lambda\left\{I(|\beta| \leq \lambda)+\frac{(a \lambda-|\beta|)_{+}}{(a-1) \lambda}(|\beta|>\lambda)\right\},
$$

with $a=3.7$ and $p_{\lambda}(0)=0$.

The DS constitutes a popular shrinkage type of variable selection method, the estimator $\hat{\boldsymbol{\beta}}$ is the solution to

$$
\min \|\hat{\boldsymbol{\beta}}\|_{1} \text { subject to }\left\|\boldsymbol{X}^{T}(\boldsymbol{y}-\boldsymbol{X} \hat{\boldsymbol{\beta}})\right\|_{\infty} \leq \delta,
$$

where $\|\hat{\boldsymbol{\beta}}\|_{1}=\sum_{j=0}^{k}\left|\hat{\beta}_{j}\right|$ is the $l_{1}$ norm, $\|\boldsymbol{a}\|_{\infty}=\max \left(\left|a_{0}\right|, \ldots,\left|a_{k}\right|\right)$ is the $l_{\infty}$ norm, and $\delta$ is a tuning constant. Phoa et al. (2009) used the DS procedure on SSDs, those factors whose coefficient estimates are greater than $\gamma$ are retained. In the simulations, we select $\gamma$ to be $1 / 10$ of the largest $\left|\hat{\beta}_{j}\right|$ in the model and choose $\delta$ according to mAIC defined in Phoa et al. 
(2009).

In this paper, factors would be classified into primary, potential, secondary groups based on their identified frequencies and the advices from experts and/or experimenters that would have a prior claim on the classification. Without the advices, we specify a factor to the primary group which is considered as active with a high possibility if its identified rate is no less than $75 \%$. Those factors that have not been identified by any method would be classified into the potential group, and the other factors whose identified rates belong to $(0,75 \%)$ constitute the secondary group. This principle is a rule of thumb, the threshold is not immutable and can be adjusted according to the experiences and background knowledge. The factors in the primary and potential groups are considered as active or inactive with a high probability, so their active performances are relatively clear compared to the factors in the secondary group which need more information to clarify.

Remark 1. It is particularly important to point out that the proposed procedure possesses great flexibility, that means the practitioners can choose the type of analysis methods, the number of analysis methods and the criteria for classifying factors in terms of their convenience, preference, experience and so on. Here we just select four particular methods to show how the proposed procedure is carried out.

\section{Methodology}

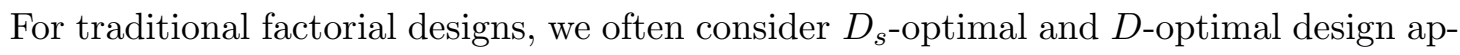
proach for follow-up experiments. If one is more interested in some parameters, i.e., a "subset" of regression parameters needs more attention, then using the $D_{s^{-}}$optimality criterion may be a better choice. Therefore, for SSDs, we would combine the $D_{s}$-optimality criterion with the Bayesian technique to give an alternative approach to the Bayesian D-optimal augmentation. 


\subsection{Bayesian $D_{s}$-optimality}

When analyzing the initial SSD, one may find that different analysis techniques may identify different sets of active factors, so it is useful to consider the results of several analysis methods (Lin, 1995). For those factors identified by one or more but not all methods applied, it is hard to assert that they are active or inactive without further information. The following example would illustrate some problems when analyzing SSDs.

Example 1. We use the $\operatorname{SSD}(7,15)$ presented in Gupta et al. (2010), four active factors are chosen randomly, and responses would be generated from the equation

$$
\boldsymbol{y}=5 \boldsymbol{x}_{3}-10 \boldsymbol{x}_{7}+2 \boldsymbol{x}_{12}+8 \boldsymbol{x}_{14}+\varepsilon, \boldsymbol{\varepsilon} \sim N\left(\mathbf{0}_{7}, \boldsymbol{I}_{7}\right) .
$$

In the model, there are two moderate effects, a large effect and a small one. The design with responses is given in Table 1. The analysis is challenging since to consider four active

Table 1: $E\left(s^{2}\right)$-optimal $\operatorname{SSD}(7,15)$ presented in Gupta et al. (2010).

\begin{tabular}{rrrrrrrrrrrrrrrrr}
\hline Run & $\boldsymbol{x}_{1}$ & $\boldsymbol{x}_{2}$ & $\boldsymbol{x}_{3}$ & $\boldsymbol{x}_{4}$ & $\boldsymbol{x}_{5}$ & $\boldsymbol{x}_{6}$ & $\boldsymbol{x}_{7}$ & $\boldsymbol{x}_{8}$ & $\boldsymbol{x}_{9}$ & $\boldsymbol{x}_{10}$ & $\boldsymbol{x}_{11}$ & $\boldsymbol{x}_{12}$ & $\boldsymbol{x}_{13}$ & $\boldsymbol{x}_{14}$ & $\boldsymbol{x}_{15}$ & $\boldsymbol{y}$ \\
\hline 1 & -1 & 1 & -1 & -1 & -1 & -1 & 1 & 1 & 1 & -1 & -1 & 1 & 1 & -1 & -1 & -21.473 \\
2 & -1 & -1 & -1 & -1 & 1 & -1 & -1 & 1 & -1 & -1 & -1 & -1 & -1 & 1 & 1 & 11.150 \\
3 & -1 & 1 & 1 & -1 & 1 & 1 & -1 & 1 & -1 & 1 & 1 & 1 & 1 & 1 & -1 & 25.483 \\
4 & 1 & -1 & -1 & 1 & -1 & -1 & -1 & -1 & -1 & 1 & -1 & 1 & 1 & 1 & -1 & 13.680 \\
5 & 1 & -1 & -1 & -1 & 1 & 1 & 1 & -1 & 1 & 1 & 1 & 1 & -1 & -1 & 1 & -22.300 \\
6 & -1 & 1 & 1 & 1 & -1 & -1 & -1 & -1 & 1 & 1 & 1 & -1 & -1 & -1 & 1 & 2.377 \\
7 & 1 & -1 & 1 & 1 & -1 & 1 & 1 & 1 & 1 & -1 & 1 & -1 & 1 & 1 & 1 & 0.265 \\
\hline
\end{tabular}

factors may violate the principle of effect sparsity (Box and Meyer, 1986). Marley and Woods (2010) pointed out that an SSD would likely to be successful under the following conditions, the factor-to-run ratio is less than two and the number of runs should beyond three times the number of active factors. Neither conditions holds for the example, however. All results from the four methods introduced in Section 2.2 are given in Table 2 , where the truly active factors are marked with a superscript "a" and "active factors" identified are indicated with a "•".

From Table 2, we can find that each method gives a different result, these four methods 
Table 2: Analysis of initial $\operatorname{SSD}(7,15)$ data.

\begin{tabular}{rrrrrrrrrrrrrrrr}
\hline Method & $\boldsymbol{x}_{1}$ & $\boldsymbol{x}_{2}$ & $\boldsymbol{x}_{3}^{\mathrm{a}}$ & $\boldsymbol{x}_{4}$ & $\boldsymbol{x}_{5}$ & $\boldsymbol{x}_{6}$ & $\boldsymbol{x}_{7}^{\mathrm{a}}$ & $\boldsymbol{x}_{8}$ & $\boldsymbol{x}_{9}$ & $\boldsymbol{x}_{10}$ & $\boldsymbol{x}_{11}$ & $\boldsymbol{x}_{12}^{\mathrm{a}}$ & $\boldsymbol{x}_{13}$ & $\boldsymbol{x}_{14}^{\mathrm{a}}$ & $\boldsymbol{x}_{15}$ \\
\hline Half Normal & & & $\bullet$ & $\bullet$ & & & $\bullet$ & & $\bullet$ & $\bullet$ & & & & $\bullet$ & $\bullet$ \\
LASSO & & & $\bullet$ & & & & $\bullet$ & & $\bullet$ & & & & $\bullet$ & $\bullet$ & $\bullet$ \\
SCAD & & & $\bullet$ & & & & $\bullet$ & & & & & $\bullet$ & & $\bullet$ & \\
DS & & & $\bullet$ & & & & $\bullet$ & & $\bullet$ & & & & & $\bullet$ & $\bullet$ \\
\hline
\end{tabular}

report nine factors of interest. According to the principle of the effect sparsity which tells that in a large set of factors, relatively few are likely to be active, it is unlikely to assert that there may have nine active factors. It is satisfying that the true active factors $\{3,7,14\}$ have been identified by all the four methods. It is disappointing that the active factor 12 has been deemed as active by only one method (SCAD). In contrast, inactive factor 9 is identified by three methods out of the four, it is not active at all. So it seems that for a factor, its identified frequency cannot tell its significance exactly and one needs more information to clarify which factors are active.

However, we can still get some useful information from the table, for example, factors $\{3,7,9,14\}$ may be very likely active since they have been selected as significant by all (or almost all) the methods. On the other hand, factors $\{1,2,5,6,8,11\}$ may be less likely active because they have not been identified by any method. For factors $\{4,10,12,13,15\}$, it is not clear whether they are active or not because they have been identified by only some methods, perhaps some are active and some are not. Based on these results, we can classify all factors into three groups: primary factors $\{3,7,9,14\}$ which are very likely to be active, potential factors $\{1,2,5,6,8,11\}$ which are likely to be inactive, and secondary factors $\{4,10,12,13,15\}$ which cannot be asserted now and need further information to clarify. Such classification results may be used as prior information for further exploration.

For the situation in Example 1, the immediate concern may be to resolve ambiguities, that is we want to confirm which factors in $\{4,10,12,13,15\}$ are active and which are not. Adding new runs to the original experiment can provide the necessary information to answer the question. Inspired by the $D_{s}$-optimality, we are more interested in those secondary terms and want to have an accurate estimate of their regression coefficients which can be achieved 
by minimizing the variances of these regression coefficients.

Assume we have classified factors into primary, potential, secondary groups and reordered them after the analysis of initial design. The primary and potential factors compose a new set "pnp" group since their active performances are relatively clear. Let

$$
\boldsymbol{X}=\left(\boldsymbol{X}_{\mathrm{pnp}}, \boldsymbol{X}_{\mathrm{sec}}\right)=\left(\begin{array}{cc}
\boldsymbol{X}_{1, \mathrm{pnp}} & \boldsymbol{X}_{1, \mathrm{sec}} \\
\boldsymbol{X}_{2, \mathrm{pnp}} & \boldsymbol{X}_{2, \mathrm{sec}}
\end{array}\right)
$$

where $\boldsymbol{X}_{\mathrm{pnp}}=\left(\boldsymbol{X}_{\mathrm{pri}}, \boldsymbol{X}_{\mathrm{pot}}\right)$ includes the intercept, $p_{1}-1$ primary terms and $p_{2}$ potential terms, $\boldsymbol{X}_{\text {sec }}$ denotes the $p_{3}$ secondary factors. Then we have

$$
\boldsymbol{X}^{T} \boldsymbol{X}=\left(\boldsymbol{X}_{\mathrm{pnp}}, \boldsymbol{X}_{\mathrm{sec}}\right)^{T}\left(\boldsymbol{X}_{\mathrm{pnp}}, \boldsymbol{X}_{\mathrm{sec}}\right)=\left(\begin{array}{cc}
\boldsymbol{X}_{\mathrm{pnp}}^{T} \boldsymbol{X}_{\mathrm{pnp}} & \boldsymbol{X}_{\mathrm{pnp}}^{T} \boldsymbol{X}_{\mathrm{sec}} \\
\boldsymbol{X}_{\mathrm{sec}}^{T} \boldsymbol{X}_{\mathrm{pnp}} & \boldsymbol{X}_{\mathrm{sec}}^{T} \boldsymbol{X}_{\mathrm{sec}}
\end{array}\right)
$$

According to the partition structure, similarly we have

$$
\boldsymbol{R}=\left(\begin{array}{cc}
\boldsymbol{R}_{\mathrm{d}} & \\
& \boldsymbol{R}_{\mathrm{sec}}
\end{array}\right)
$$

where

$$
\boldsymbol{R}_{\mathrm{pnp}}=\left(\begin{array}{cc}
\mathbf{0}_{p_{1} \times p_{1}} & \mathbf{0}_{p_{1} \times p_{2}} \\
\mathbf{0}_{p_{2} \times p_{1}} & \boldsymbol{I}_{p_{2}} / \tau^{2}
\end{array}\right)
$$

and $\boldsymbol{R}_{\mathrm{sec}}=\boldsymbol{I}_{p_{3}} / \gamma^{2}$. Thus the posterior variance for $\boldsymbol{\beta}$ can be rewritten as

$$
\sigma^{2}\left(\boldsymbol{X}^{T} \boldsymbol{X}+\boldsymbol{R}\right)^{-1}=\sigma^{2}\left(\begin{array}{cc}
\boldsymbol{X}_{\mathrm{pnp}}^{T} \boldsymbol{X}_{\mathrm{pnp}}+\boldsymbol{R}_{\mathrm{pnp}} & \boldsymbol{X}_{\mathrm{pnp}}^{T} \boldsymbol{X}_{\mathrm{sec}} \\
\boldsymbol{X}_{\mathrm{sec}}^{T} \boldsymbol{X}_{\mathrm{pnp}} & \boldsymbol{X}_{\mathrm{sec}}^{T} \boldsymbol{X}_{\mathrm{sec}}+\boldsymbol{R}_{\mathrm{sec}}
\end{array}\right)^{-1}
$$

so the lower right $p_{3} \times p_{3}$ submatrix of $\left(\boldsymbol{X}^{T} \boldsymbol{X}+\boldsymbol{R}\right)^{-1}$ can be shown to be

$$
\left[\boldsymbol{X}_{\mathrm{sec}}^{T} \boldsymbol{X}_{\mathrm{sec}}+\boldsymbol{R}_{\mathrm{sec}}-\boldsymbol{X}_{\mathrm{sec}}^{T} \boldsymbol{X}_{\mathrm{pnp}}\left(\boldsymbol{X}_{\mathrm{pnp}}^{T} \boldsymbol{X}_{\mathrm{pnp}}+\boldsymbol{R}_{\mathrm{pnp}}\right)^{-1} \boldsymbol{X}_{\mathrm{pnp}}^{T} \boldsymbol{X}_{\mathrm{sec}}\right]^{-1}
$$


According to the $D_{s}$-optimality, the best choice of $\boldsymbol{X}_{2}$ is one that maximizes

$$
\left|\boldsymbol{X}_{\mathrm{sec}}^{T} \boldsymbol{X}_{\mathrm{sec}}+\boldsymbol{R}_{\mathrm{sec}}-\boldsymbol{X}_{\mathrm{sec}}^{T} \boldsymbol{X}_{\mathrm{pnp}}\left(\boldsymbol{X}_{\mathrm{pnp}}^{T} \boldsymbol{X}_{\mathrm{pnp}}+\boldsymbol{R}_{\mathrm{pnp}}\right)^{-1} \boldsymbol{X}_{\mathrm{pnp}}^{T} \boldsymbol{X}_{\mathrm{sec}}\right|,
$$

where

$$
\begin{aligned}
\boldsymbol{X}_{\mathrm{sec}}^{T} \boldsymbol{X}_{\mathrm{sec}} & =\boldsymbol{X}_{1, \mathrm{sec}}^{T} \boldsymbol{X}_{1, \mathrm{sec}}+\boldsymbol{X}_{2, \mathrm{sec}}^{T} \boldsymbol{X}_{2, \mathrm{sec}} \\
\boldsymbol{X}_{\mathrm{sec}}^{T} \boldsymbol{X}_{\mathrm{pnp}} & =\boldsymbol{X}_{1, \mathrm{sec}}^{T} \boldsymbol{X}_{1, \mathrm{pnp}}+\boldsymbol{X}_{2, \mathrm{sec}}^{T} \boldsymbol{X}_{2, \mathrm{pnp}} \\
\boldsymbol{X}_{\mathrm{pnp}}^{T} \boldsymbol{X}_{\mathrm{pnp}} & =\boldsymbol{X}_{1, \mathrm{pnp}}^{T} \boldsymbol{X}_{1, \mathrm{pnp}}+\boldsymbol{X}_{2, \mathrm{pnp}}^{T} \boldsymbol{X}_{2, \mathrm{pnp}}
\end{aligned}
$$

Similar to Gutman et al. (2014), the final design can be called the Bayesian $D_{s}$-optimal augmented design.

In fact, for Bayesian $D$-optimality, maximizing $\left|\boldsymbol{X}^{T} \boldsymbol{X}+\boldsymbol{R}\right|$ is in some sense equivalent to minimizing the correlations between all factors under some prior information which is referred by the matrix $R$. In addition, with the knowledge of the block matrix, we have

$\left|\boldsymbol{X}^{T} \boldsymbol{X}+\boldsymbol{R}\right|=\left|\boldsymbol{X}_{\mathrm{pnp}}^{T} \boldsymbol{X}_{\mathrm{pnp}}+\boldsymbol{R}_{\mathrm{pnp}}\right| \cdot\left|\boldsymbol{X}_{\mathrm{sec}}^{T} \boldsymbol{X}_{\mathrm{sec}}+\boldsymbol{R}_{\mathrm{sec}}-\boldsymbol{X}_{\mathrm{sec}}^{T} \boldsymbol{X}_{\mathrm{pnp}}\left(\boldsymbol{X}_{\mathrm{pnp}}^{T} \boldsymbol{X}_{\mathrm{pnp}}+\boldsymbol{R}_{\mathrm{pnp}}\right)^{-1} \boldsymbol{X}_{\mathrm{pnp}}^{T} \boldsymbol{X}_{\mathrm{sec}}\right|$,

which is Eq. (1) multiplied by $\left|\boldsymbol{X}_{\mathrm{pnp}}^{T} \boldsymbol{X}_{\mathrm{pnp}}+\boldsymbol{R}_{\mathrm{pnp}}\right|$. Thus for Bayesian $D_{s}$-optimality, it focuses more attention on the correlations involving secondary factors, the benefit is the reduction in correlation relating to secondary terms and that would make picking out active factors from the secondary set be easier.

\subsection{Adding a blocking factor}

In practice, there could be a long period of time between the original experiment and the follow up experiment. So it is common to observe a shift in average responses between the two experiments (Goos et al. 2011). Therefore it would be better to add a term, a blocking 
factor $\boldsymbol{x}_{p+1}$, to capture this potential shift in the mean response,

$$
\boldsymbol{y}=\boldsymbol{X} \boldsymbol{\beta}+\boldsymbol{x}_{p+1} \delta+\varepsilon
$$

where the parameter $\delta$ represents the shift in responses, and the factor $\boldsymbol{x}_{p+1}$ is a 2-level factor that takes the value +1 for all the runs in the original experiment and -1 for all the runs in the follow-up experiment. In general, we call the parameter $\delta$ a blocking effect.

For the original experiment, the new model matrix is $\left(\boldsymbol{X}_{1}, \mathbf{1}_{n_{1}}\right)$, we know that the first column of $\boldsymbol{X}_{1}$ is equal to $\mathbf{1}_{n_{1}}$, thus the blocking effect is confounded with the intercept. If we have run the follow-up experiments, then the new complete model matrix is

$$
\boldsymbol{X}_{\mathrm{c}}=\left(\begin{array}{cc}
\boldsymbol{X}_{1} & \mathbf{1}_{n_{1}} \\
\boldsymbol{X}_{2} & -\mathbf{1}_{n_{2}}
\end{array}\right)
$$

Let the prior distribution of the parameters be $\boldsymbol{\beta}_{\mathrm{c}} \mid \sigma^{2} \sim N\left(\boldsymbol{\beta}_{\mathrm{c} 0}, \sigma^{2} \boldsymbol{R}_{\mathrm{c}}^{-1}\right)$, where $\boldsymbol{\beta}_{\mathrm{c}}=$ $\left(\boldsymbol{\beta}^{T}, \delta\right)^{T}, \boldsymbol{R}_{\mathrm{c}}$ is a prior variance with

$$
\boldsymbol{R}_{\mathrm{c}}=\left(\begin{array}{cc}
\boldsymbol{R} & \mathbf{0}_{p} \\
\mathbf{0}_{p}^{T} & 1 / \xi^{2}
\end{array}\right)
$$

Assume the conditional distribution is $\boldsymbol{y} \mid\left(\boldsymbol{\beta}_{\mathrm{c}}, \sigma^{2}\right) \sim N\left(\boldsymbol{X}_{\mathrm{c}} \boldsymbol{\beta}_{\mathrm{c}}, \sigma^{2} \boldsymbol{I}_{n_{1}+n_{2}}\right)$, then posterior distribution for $\boldsymbol{\beta}_{\mathrm{c}}$ given $\boldsymbol{y}$ is $\boldsymbol{\beta}_{\mathrm{c}} \mid \boldsymbol{y} \sim N\left(\boldsymbol{b}_{\mathrm{c}}, \sigma^{2}\left(\boldsymbol{X}_{\mathrm{c}}^{T} \boldsymbol{X}_{\mathrm{c}}+\boldsymbol{R}_{\mathrm{c}}\right)^{-1}\right)$, where $\boldsymbol{b}_{\mathrm{c}}=\left(\boldsymbol{X}_{\mathrm{c}}^{T} \boldsymbol{X}_{\mathrm{c}}+\right.$ $\left.\boldsymbol{R}_{\mathrm{c}}\right)^{-1}\left(\boldsymbol{X}_{\mathrm{c}}^{T} \boldsymbol{y}+\boldsymbol{R}_{\mathrm{c}} \boldsymbol{\beta}_{\mathrm{c} 0}\right)$ and

$$
\boldsymbol{X}_{\mathrm{c}}^{T} \boldsymbol{X}_{\mathrm{c}}=\left(\begin{array}{cc}
\boldsymbol{X}_{1}^{T} \boldsymbol{X}_{1}+\boldsymbol{X}_{2}^{T} \boldsymbol{X}_{2} & \boldsymbol{X}_{1}^{T} \mathbf{1}_{n_{1}}-\boldsymbol{X}_{2}^{T} \mathbf{1}_{n_{2}} \\
\mathbf{1}_{n_{1}}^{T} \boldsymbol{X}_{1}-\mathbf{1}_{n_{2}}^{T} \boldsymbol{X}_{2} & n_{1}+n_{2}
\end{array}\right)
$$

Similar to the Bayesian $D$-optimal designs, if we aim to reduce the error variances of the parameter estimators, $\operatorname{Var}\left(\boldsymbol{b}_{\mathrm{c}}\right)=\sigma^{2}\left(\boldsymbol{X}_{\mathrm{c}}^{T} \boldsymbol{X}_{\mathrm{c}}+\boldsymbol{R}_{\mathrm{c}}\right)^{-1}$, the optimal augmented SSDs should be 
constructed to maximize

$$
\left|\boldsymbol{X}_{\mathrm{c}}^{T} \boldsymbol{X}_{\mathrm{c}}+\boldsymbol{R}_{\mathrm{c}}\right|=\left|\begin{array}{cc}
\boldsymbol{X}_{1}^{T} \boldsymbol{X}_{1}+\boldsymbol{X}_{2}^{T} \boldsymbol{X}_{2}+\boldsymbol{R} & \boldsymbol{X}_{1}^{T} \mathbf{1}_{n_{1}}-\boldsymbol{X}_{2}^{T} \mathbf{1}_{n_{2}}+\mathbf{0}_{p} \\
\mathbf{1}_{n_{1}}^{T} \boldsymbol{X}_{1}-\mathbf{1}_{n_{2}}^{T} \boldsymbol{X}_{2}+\mathbf{0}_{p}^{T} & n_{1}+n_{2}+1 / \xi^{2}
\end{array}\right|
$$

Thus, in term of the Bayesian D-optimality, we would maximize

$$
\left|\boldsymbol{X}_{1}^{T} \boldsymbol{X}_{1}+\boldsymbol{X}_{2}^{T} \boldsymbol{X}_{2}+\boldsymbol{R}-\frac{1}{n_{1}+n_{2}+1 / \xi^{2}}\left(\boldsymbol{X}_{1}^{T} \mathbf{1}_{n_{1}}-\boldsymbol{X}_{2}^{T} \mathbf{1}_{n_{2}}\right)\left(\mathbf{1}_{n_{1}}^{T} \boldsymbol{X}_{1}-\mathbf{1}_{n_{2}}^{T} \boldsymbol{X}_{2}\right)\right|,
$$

to get $\boldsymbol{X}_{2}$ generally, since $\left|\boldsymbol{X}_{\mathrm{c}}^{T} \boldsymbol{X}_{\mathrm{c}}+\boldsymbol{R}_{\mathrm{c}}\right|$ equals Eq.(3) multiplied by $\left(n_{1}+n_{2}+1 / \xi^{2}\right)$ according to properties of the block matrix.

When analyzing the augmented design, the blocking factor would be classified as a secondary term since one cannot detect the blocking effect with the initial design and we are not sure if there is a mean shift between the two experiments. This is different with Jones et al. (2008) where they designated the blocking factor as a primary effect. For convenience, we would set $\xi^{2}=\gamma^{2}$ here. It is easy to consider the Bayesian $D_{s^{-}}$optimal augmented SSDs since we just need to put the blocking factor into the secondary group with an appropriate adjustment for $\boldsymbol{X}$ and $\boldsymbol{R}$.

A coordinate-exchange algorithm would be used to construct the optimal augmented SSDs here, more detailed discussion of the algorithm can be found in Goos et al. (2011).

Example 2. To visually display how a neglected active blocking factor affect the augmented design and the factors' identification, consider the $\operatorname{SSD}(7,15)$ in Table 1 with responses generated from the equation

$$
\boldsymbol{y}^{*}=5 \boldsymbol{x}_{3}-10 \boldsymbol{x}_{7}+2 \boldsymbol{x}_{12}+8 \boldsymbol{x}_{14}+\delta \boldsymbol{x}_{16}+\boldsymbol{\varepsilon}, \boldsymbol{\varepsilon} \sim N\left(\mathbf{0}_{7}, \boldsymbol{I}_{7}\right)
$$

where $\boldsymbol{x}_{16}=1$ for the first seven runs and $\boldsymbol{x}_{16}=-1$ for the added $n_{2}$ runs, and $\delta$ denotes the blocking effect. Note that the $\boldsymbol{y}^{*}$ in Eq. (4) is a little different from that of Example 1 because we have added a blocking factor here to describe the mean shift between two stages. However the factors picked out would not change since the mean shift and the former intercept 
are confounded as a new intercept parameter when analyzing the initial design $\operatorname{SSD}(7,15)$. The factors have been classified into three groups: primary factors $3,7,9,14$, potential factors $1,2,5,6,8,11$, and the others are secondary factors.

All examples in this paper use $\gamma^{2}=100$ and $\tau^{2}=5$, the same with that of Gutman et al. (2014). Based on the classification, we use the coordinate-exchange algorithm with 2000 initial random designs to add $n_{2}=4$ runs under the Bayesian $D_{s}$-optimality, and the setting for the number of initial random designs comes from the simulation discussed later. Ignoring or considering the blocking effect, we get two Bayesian $D_{s}$-optimal augmented designs: $\operatorname{SSD}(11,15)_{1}$ whose added runs are given in the upper part of Table 3 and $\operatorname{SSD}(11,15)_{2}$ whose added runs are in the lower part of Table 3. Note that for both augmented SSDs, all settings are the same in the progress of constructing the optimal follow-up runs except that the blocking factor, $\boldsymbol{x}_{16}=(1,1,1,1,1,1,1,-1,-1,-1,-1)^{\prime}$, has been put in the secondary group for the latter.

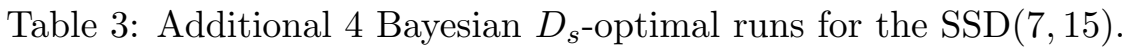

\begin{tabular}{lcrrrrrrrrrrrrrrrr}
\hline & Run & $\boldsymbol{x}_{1}$ & $\boldsymbol{x}_{2}$ & $\boldsymbol{x}_{3}$ & $\boldsymbol{x}_{4}$ & $\boldsymbol{x}_{5}$ & $\boldsymbol{x}_{6}$ & $\boldsymbol{x}_{7}$ & $\boldsymbol{x}_{8}$ & $\boldsymbol{x}_{9}$ & $\boldsymbol{x}_{10}$ & $\boldsymbol{x}_{11}$ & $\boldsymbol{x}_{12}$ & $\boldsymbol{x}_{13}$ & $\boldsymbol{x}_{14}$ & $\boldsymbol{x}_{15}$ & $\boldsymbol{y}^{*}$ \\
\hline \multirow{3}{*}{ Ignoring } & 8 & 1 & 1 & 1 & 1 & -1 & 1 & -1 & -1 & -1 & -1 & 1 & 1 & -1 & 1 & 1 & 20.190 \\
blocking & 9 & 1 & -1 & -1 & 1 & 1 & 1 & 1 & -1 & -1 & 1 & 1 & -1 & -1 & -1 & -1 & -29.880 \\
& 10 & 1 & 1 & 1 & -1 & -1 & 1 & -1 & -1 & 1 & -1 & 1 & -1 & -1 & 1 & -1 & 16.751 \\
& 11 & 1 & 1 & 1 & -1 & -1 & 1 & -1 & -1 & -1 & 1 & 1 & -1 & 1 & 1 & 1 & 18.222 \\
\hline \hline \multirow{3}{*}{ Considering } & 8 & 1 & -1 & 1 & 1 & -1 & 1 & 1 & 1 & -1 & -1 & 1 & 1 & -1 & 1 & 1 & 2.176 \\
blocking & 10 & 1 & -1 & 1 & -1 & -1 & 1 & 1 & 1 & 1 & 1 & 1 & -1 & -1 & 1 & -1 & -2.441 \\
& 11 & -1 & -1 & -1 & 1 & 1 & -1 & -1 & 1 & 1 & 1 & -1 & 1 & 1 & 1 & 1 & 10.244 \\
\hline
\end{tabular}

Figure 1 shows the correlation maps for both $\operatorname{SSD}(11,15)$ 's. In the figure, "Vi" means the $i$ th factor for $i=1, \ldots, 15$ and "block" denotes the blocking factor, in addition, a light color represents a weak correlation between factors, while a black color represents a high correlation. To make the graphical representation clear, the diagonal elements have been set to blank in the figure though their values should be 1 . The marked square box in the diagram corresponds to the "pnp" group including primary and potential factors, secondary and blocking factors stand back, the order is the same with the above. Such an arrangement 


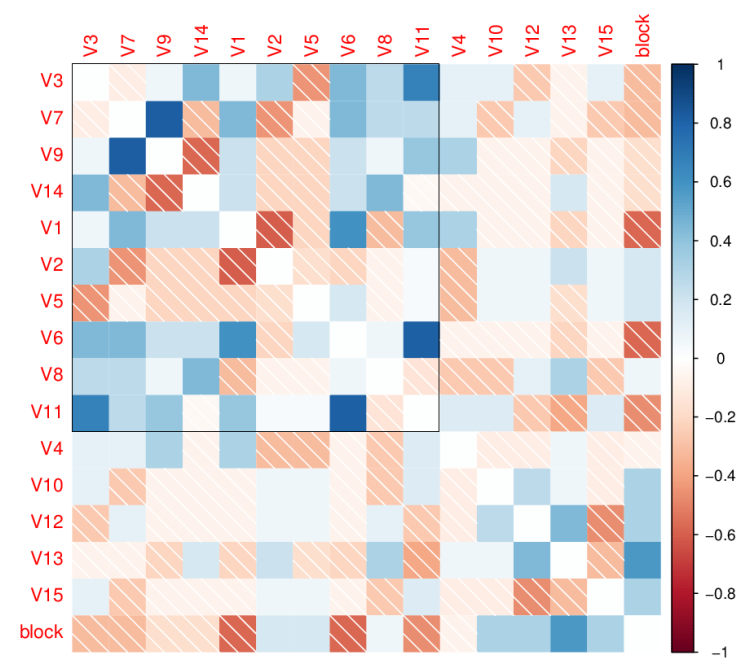

(a) $\operatorname{SSD}(11,15)_{1}$

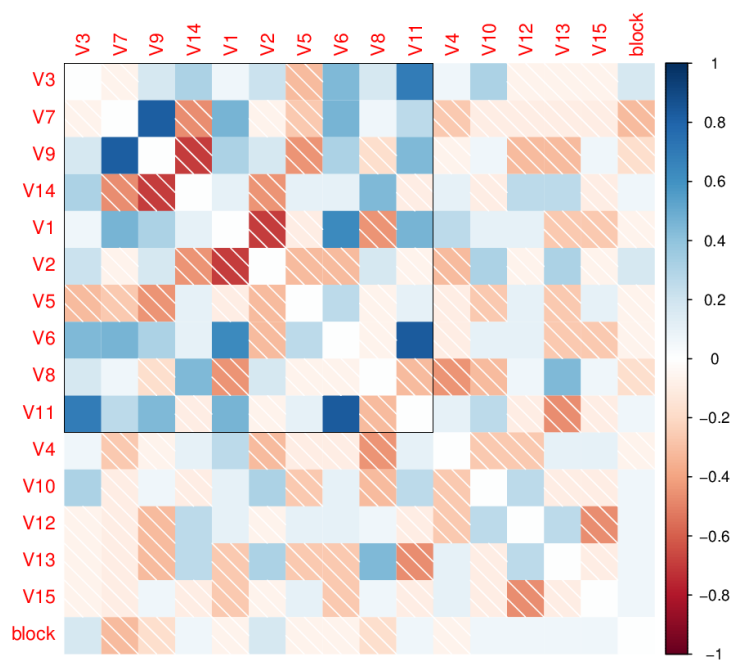

(b) $\operatorname{SSD}(11,15)_{2}$

Figure 1: Correlation maps for both augmented SSD(11,15)'s

is made for displaying the correlations among two classes of factors.

Both Bayesian $D_{s}$-optimal augmented designs behave similarly for the experimental factors. For each SSD, it is apparent that the color in the marked box is darker than in the other area, so correlations among factors in the "pnp" group are relatively stronger than those involving secondary factors, thereby increasing the likelihood for an active factor to be identified from the secondary group. This performance coincides considerably with the explantation given at the end of Section 3.1.

The last columns in the maps display differently, the color of $\operatorname{SSD}(11,15)_{2}$ is much lighter, which means smaller correlations between the blocking factor and the other experimental factors. For $\operatorname{SSD}(11,15)_{1}$, three factors, $x_{1}, x_{6}, x_{13}$, are the most relevant to the blocking factor and their absolute values of correlation coefficients are all 0.5714 , the high correlations will make them to be incorrectly identified as active easily when the blocking effect is truly active, see the following Table 4 . However. for $\operatorname{SSD}(11,15)_{2}$, only factor $x_{7}$ has a relatively large correlation with the blocking factor and the correlation coefficient is -0.3105 .

Consider there is a moderate blocking effect, say $\delta=4$. Now the present responses $\boldsymbol{y}^{*}$ for the initial design $\operatorname{SSD}(7,15)$ are the sum of $\delta$ and $\boldsymbol{y}$ in Table 1 . For the added runs, 
their responses are generated from Eq.(4) and listed in Table 3 . Four methods introduced in Section 2.2 are used to identify the active effects for both augmented SSDs and the results are displayed in Table 4 . For $\operatorname{SSD}(11,15)_{1}$, two different ways of dealing with the blocking effect are considered: ignoring or including it in the model, so the first two parts in Table 4 are both for $\operatorname{SSD}(11,15)_{1}$.

Table 4: Analysis of $\boldsymbol{y}^{*}$ on the Bayesian $D_{s}$-optimal augmented $\operatorname{SSD}(11,15)$.

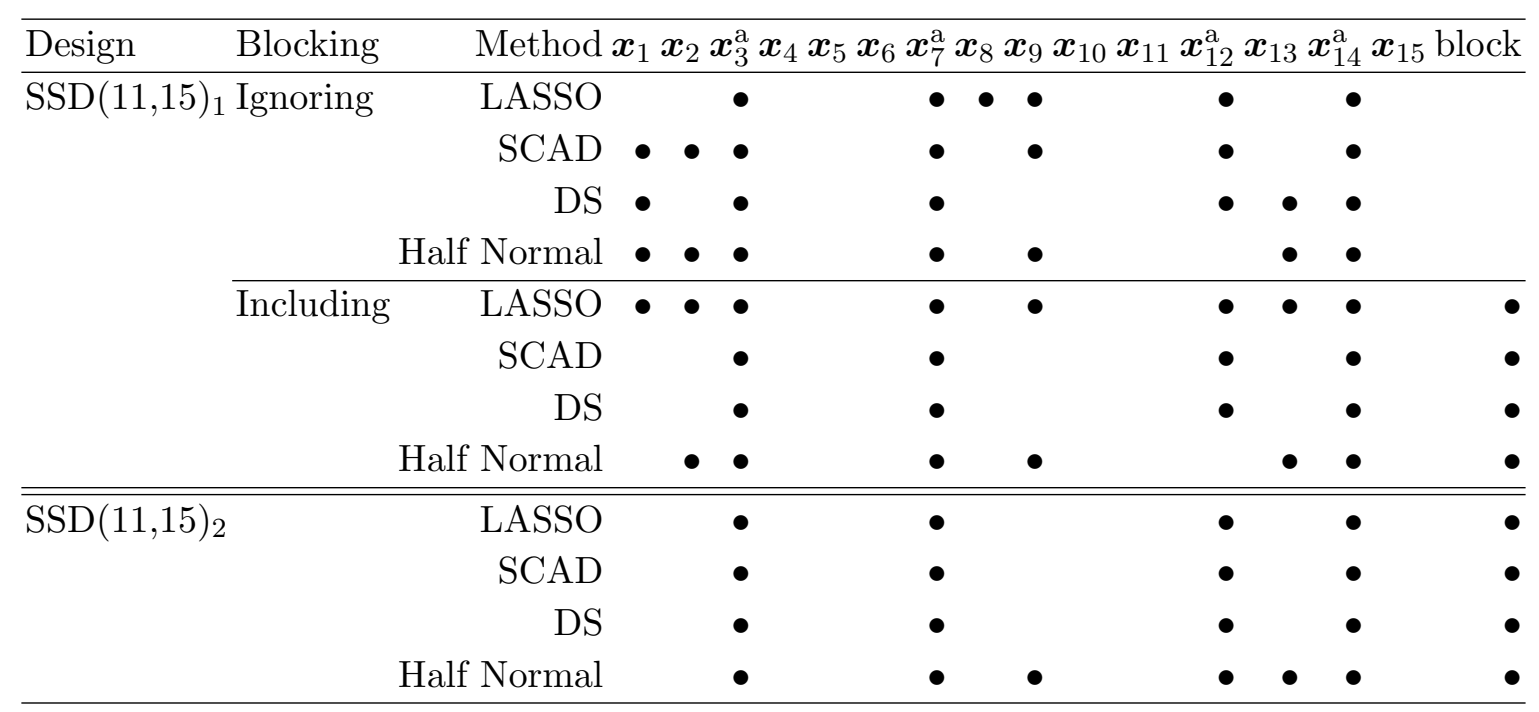

Firstly, all active factors are identified by at least three out of four methods successfully, especially for $\operatorname{SSD}(11,15)_{2}$, each active factor can be identified by four methods. If the blocking effect is included in the model, both SSDs can identify the blocking effect though the blocking factor is not considered when constructing the follow-up runs for $\operatorname{SSD}(11,15)_{1}$. However, for $\operatorname{SSD}(11,15)_{1}$, some inactive factors have also been incorrectly picked up as active factors by at least one analysis method. Ignoring the blocking effect when modeling the data will produce a higher Type I error than including it in the model. Furthermore, even we include the blocking effect in the model, the performance of $\operatorname{SSD}(11,15)_{1}$ is still not so good as the $\operatorname{SSD}(11,15)_{2}$ in terms of the analysis results because of the high correlations between the experimental factors and the blocking factor in $\operatorname{SSD}(11,15)_{1}$. So if a truly active blocking factor is not taken into account when constructing the follow-up runs, there may be relatively large correlations between the experimental factors and the blocking factor in the augmented SSDs and this point will result in a high type I error. For example, for $\operatorname{SSD}(11,15)_{1}$, the 
inactive factors $\boldsymbol{x}_{1}$ has been selected as active incorrectly by three out of four methods, note the correlation coefficient between it and the blocking factor is 0.5714 , another correlated inactive factor $\boldsymbol{x}_{13}$ has also been selected as active by two methods.

\section{$4 \quad$ Simulation studies and results}

In the section, simulations are carried out to study the performance of two classes of augmented Bayesian $D$-optimal and Bayesian $D_{s}$-optimal SSDs, some comparisons are also made.

\subsection{Features varied}

We vary the following simulation settings in our study.

1. Factor-to-run ratios in the SSDs. Three choices of decreasing difficulty would be used: $\operatorname{SSD}(7,15), \operatorname{SSD}(8,13)$ and $\operatorname{SSD}(9,12)$, with the ratios varying from 2.14 to 1.33 . Three initial SSDs are all $E\left(s^{2}\right)$-optimal designs, where the first two come from Gutman et al. (2014) and the last one is constructed using the nonorthogonal array algorithm of Nguyen (1996) in Gendex (http://www.designcomputing.net/gendex/). The reason to consider such SSDs with few runs is that the commonly used methods do not perform well for these designs, so there may be an urgent need to add trials to clarify which factors are active.

2. Experimental scenarios. We vary the number and magnitude of active factors over three scenarios. Specifically, the magnitude of the regression coefficient for each of the $a$ active factors and one blocking factor is drawn from $N(\mu, 1)$, with different scenarios of :
(1) $a=3, \mu=5$;
(2) $a=4, \mu=4$;
(3) $a=5, \mu=3$.

3. Design augmenting criteria. Here we include the Bayesian $D$-optimal and the new Bayesian $D_{s}$-optimal augmented SSDs. 
4. The number of added runs $n_{2}$. We choose to add $n_{2}=2,3, \ldots, k+1-n_{1}$ follow-up runs, the augmented design would not be supersaturated any more when $n_{2}>k+1-n_{1}$.

5. The Bayesian $D$-optimal and Bayesian $D_{s}$-optimal augmented SSDs are all generated using coordinate-exchange algorithm with $M$ random starts.

We have made a number of simulations to find how many random-start designs would present an optimal or near optimal augmented SSDs, i.e., the values of the optimal criterion in Eq.(1) (or Eq.(3)) would have no or very small difference. As expected, an appropriate choice is related to the number of added runs, the number of factors, and the classification of factors. For instance, in Example 2, when one run is added, 50 random starts are enough to give a Bayesian $D_{s}$-optimal augmented $\operatorname{SSD}(7+1,15)$ since the results with $M=50$ and the results with $M=500$ are the same. However, when adding $n_{2}=3$ runs, there are some differences in the values of criterion (1), even we choose 100 random starts. A large number of simulations show that the the criterion value is relatively stable only when $M$ is around or above 1000. As the number of added runs increases, the value of Eq.(1) increases gradually, meanwhile, much more start designs are needed to get an optimal or near optimal augmented SSD. However, in general, when $n_{2}$ is larger, even if the values of Eq.(1) (or Eq.(3)) are slightly different for two augmented SSDs, the impact on the latter data analysis is very small. Thus we set $M=\left\lceil n_{2} / 2\right\rceil * 1000$, where $\lceil\cdot\rceil$ is for rounding up, and it would present a near optimal augmented SSD based on our simulation results.

In each of the 1000 iterations:

1. Among the $2 \mathrm{nd}, 3 \mathrm{rd}, \ldots, k+1$ th columns of $\boldsymbol{X}, a$ columns are randomly assigned as the active experimental factors. Their coefficients $\boldsymbol{\beta}$ and the coefficient $\delta$ for the blocking factor are obtained by sampling from $N(\mu, 1)$ and randomly assigning signs $(+$ or -$)$.

2. For simplicity, the remaining $k-a$ columns (inactive effects) are assigned to be exactly zero.

3. The response vector is generated from the model in Eq. (2) with error $\varepsilon \sim N\left(\mathbf{0}_{n_{1}}, \boldsymbol{I}_{n_{1}}\right)$.

4. All factors would be classified into their appropriate groups in terms of the classifying 
principle after being analyzed by four model selection methods introduced in Section 2.2. The blocking factor is assigned as secondary term.

5. Add $n_{2}$ runs by maximizing the objective functions in Eq.(1) and Eq.(3) to get the Bayesian $D_{s^{-}}$optimal and the Bayesian $D$-optimal augmented SSDs respectively.

6. Generate the new responses for the added $n_{2}$ runs. Analyze the final augmented SSDs with four model selection methods. Then those factors (identified as active by at least three methods here) classified into the primary group are the declared "active" factors now.

\subsection{Simulation results for augmenting SSDs}

Three different criteria similar to Marley and Woods (2010) would be used to assess the performance of the augmented designs.

Power: The average proportion of active factors classified into the primary group.

Type I error: The average proportion of the inactive factors classified into the primary group.

Coverage: The average proportion of times that all the truly active effects are classified into the primary group.

Clearly, Power and Coverage are the-larger-the-better, and Type I error is the-smaller-thebetter. Figures 24 show the Power, Type I error and Coverage for three SSDs, respectively.

For the $\operatorname{SSD}\left(7+n_{2}, 15\right)$, Figure 2 shows the dominance of the Bayesian $D_{s}$-optimal augmented SSDs with regard to Power, where the superiority is particularly evident when adding fewer runs. For example, in the first scenario, $a=3, \mu=5$, when $n_{2}=2$, the difference of Power between the two classes of augmented SSDs is close to 0.1, however the difference is very small when adding eight runs. Also, the Bayesian $D_{s}$-optimal augmented SSDs have slightly higher coverage proportions. Note that two classes of augmenting strategies have similar performance with respect to Type I error. Thus Bayesian $D_{s}$-optimality criterion 

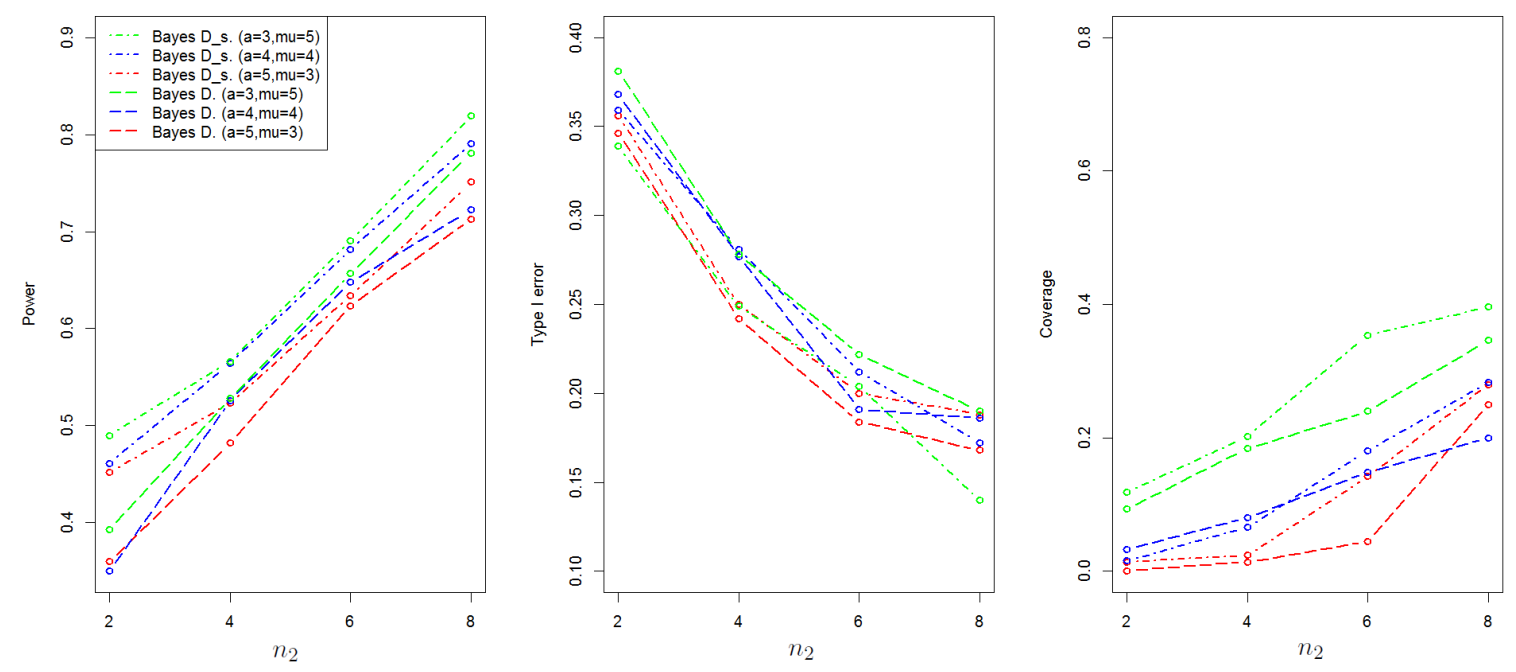

Figure 2: Performance for two classes of augmented $\operatorname{SSD}\left(7+n_{2}, 15\right)$ 's

is recommended if one can afford few runs because it would produce a more economical augmented SSD with a good performance.

Not surprisingly, as the number of adding runs increases, both classes of the augmented designs perform well. Generally, when adding the same runs, the augmented SSDs have consistently worse performance for harder scenarios in terms of all three measures. Note that for both classes of augmented SSDs, we have low coverage proportions relative to the good performance on the Power. This means some small effects may not be identified though multiple runs have been added. In fact, most of the missing active factors are the members that cannot be identified by any method for the initial designs. So for those small effects which cannot be selected as active by at least one of four methods applied, to identify all of them is still difficult even adding much more runs.

For two classes of $\operatorname{SSD}\left(8+n_{2}, 13\right)$, Figure 3 shows that the Bayesian $D_{s}$-optimal criterion leads to a bit higher Power and Coverage although the differences are not large. Also, the comparisons indicate that the Bayesian $D_{s}$-optimal augmented SSDs have little lower Type I error rates. For the third scenario, it seems that the Bayesian $D_{s}$-optimal augmented SSDs perform better than the Bayesian $D$-optimal augmented SSDs in terms of the three measures. In addition, the last scenario clearly has the worst performance comparing to the first two 

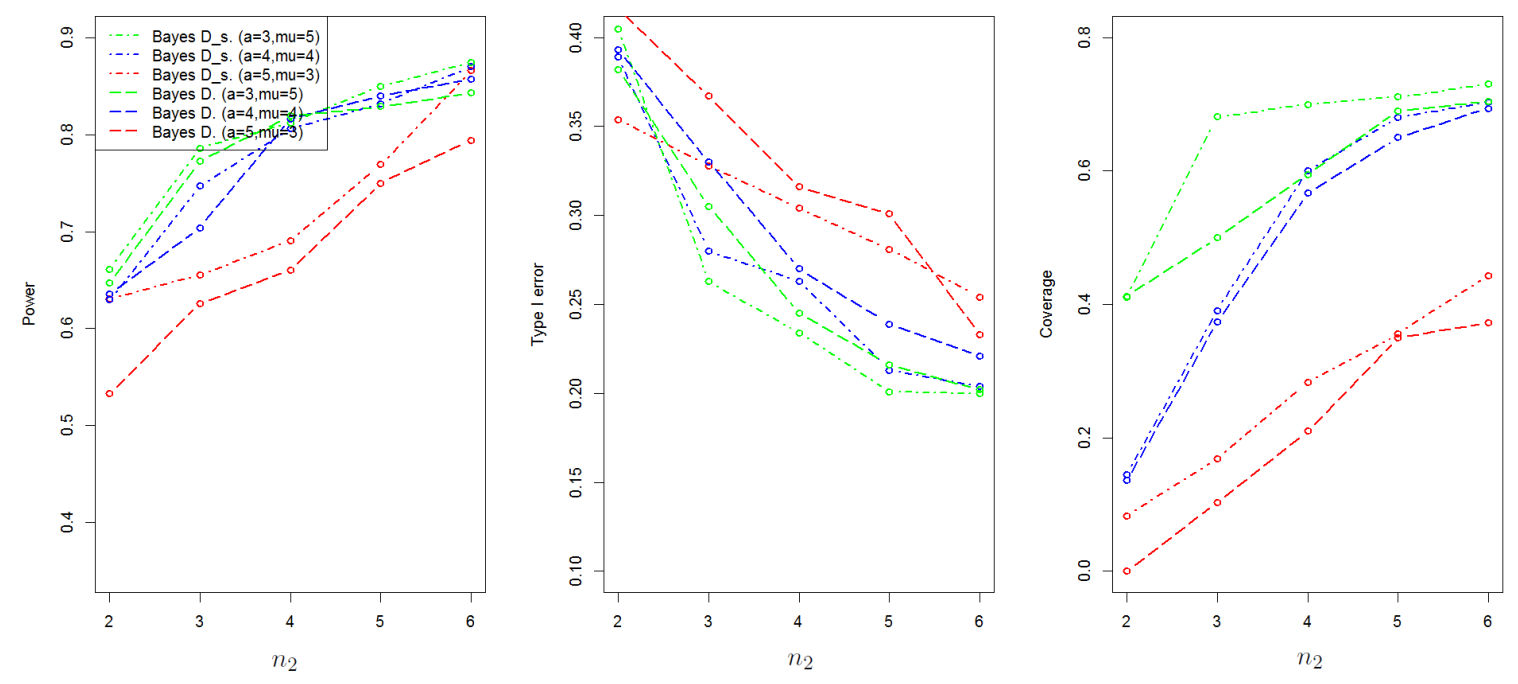

Figure 3: Performance for two classes of augmented $\operatorname{SSD}\left(8+n_{2}, 13\right)$ 's
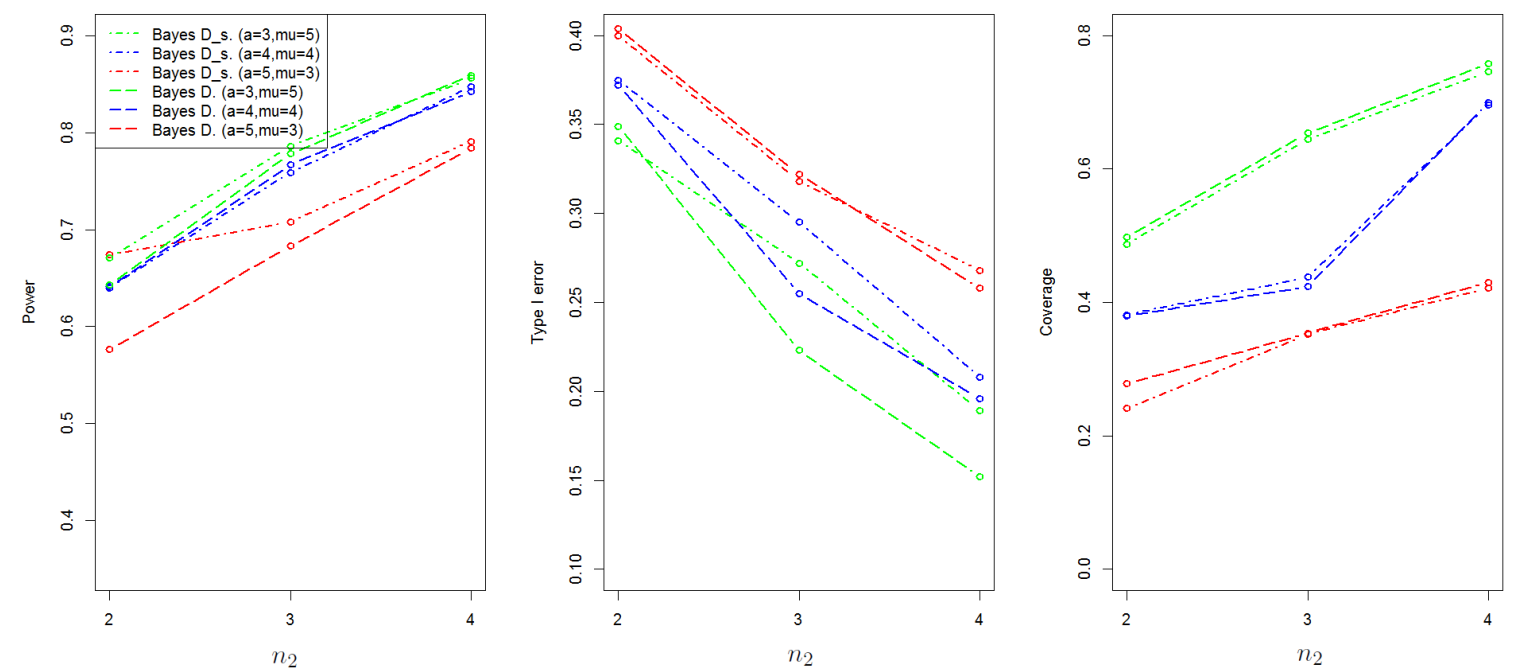

Figure 4: Performance for two classes of augmented $\operatorname{SSD}\left(9+n_{2}, 12\right)$ 's

scenarios. Note that for $\operatorname{SSD}\left(7+n_{2}, 15\right)$, the settings of the three scenarios have some impacts on the performance, but not so large as that for $\operatorname{SSD}\left(8+n_{2}, 13\right)$. Based on the simulations for $\operatorname{SSD}\left(7+n_{2}, 15\right)$ and $\operatorname{SSD}\left(8+n_{2}, 13\right)$, it seems that the Bayesian $D_{s^{-o p t i m a l}}$ augmenting criterion tends to produce designs with higher Power and Coverage, and lower Type I error rates for the hard settings. 
For the $\operatorname{SSD}\left(9+n_{2}, 12\right)$, two classes of augmented SSDs perform well as expected since the analysis of initial design can present a good classification for the factors, that is about $50 \%$ active factors are specified to the primary group and only about $10 \%$ active factors are incorrectly classified into the potential group. Here, the Bayesian $D_{s}$-optimal augmented SSDs do not outperform the Bayesian $D$-optimal augmented SSDs any more, i.e., two classes of augmentation methods present similar results in terms of the three evaluation measures.

Through the above simulations, we know that for the initial SSDs with high factor-torun ratios, the identification results from various methods are different. When adding fewer runs are considered, employing the Bayesian $D_{s}$-optimal augmented design would be a better choice. Even the factor-to-run ratio is not very high, if there are great differences in the results of various methods for the initial SSDs, this performance suggests us that the number of active factors may be a litter large or there are some small effects, the Bayesian $D_{s}$-optimal augmented design should be the preference too.

Remark 2. When adding follow-up experiments, the practitioners are very concerned about the practical question, given $n_{1}$ what will be an ideal value for $n_{2}$ ? For traditional SSDs, Marley and Woods (2010) concluded that the number of all runs should be at least three times the number of active effects. However, it is not easy to answer this question for the augmented SSDs since it would involve the objective of experiment, the correlations in the initial design model, the complexity of the model and so on. In our simulation, we have considered all the values of $n_{2} \in\left\{2, \ldots, k+1-n_{1}\right\}$, though not all results are presented for $\operatorname{SSD}(7,15)$ since Figure 2 can still clearly show the trend of increasing or decreasing for the three measures. We find that for initial SSDs with moderate factor-to-run ratios, such as $\operatorname{SSD}(9,12)$ and $\operatorname{SSD}(8,13)$, adding $n_{2}=k+1-n_{1}$ runs is enough to present a good augmented SSD if the model does not include many small active effects. When an SSD with a high factor-to-run ratio is used in the first stage experiment, difficulty to identify those relatively small effects would result in an inaccurate factor classification and eventually lead to a bad result, though the result is somewhat meaningful. In general, the purpose of using an SSD is just to find the important factors. 


\section{Concluding remarks}

SSDs are typically employed to screen a few factors from many candidates and they have great potential to aid in discoveries in a resource-efficient manner. However, screening experiments often leave some questions unanswered and adding new runs to the original experiment should allow us to clear up our uncertainty. In this paper, we have presented the Bayesian $D_{s}$-optimality to add runs to existing SSDs by using information from the initial experiment. The simulation studies in Section 4 indicate that the augmentation strategy performs well, especially when we have a proper prior classification for the factors, most of the active factors and the mean shift can be identified with adding a few runs. Even for those challenging settings, the factor classification is not so accurate because of the complicated confounding pattern in the initial design, the analysis results of the final augmented SSDs are still pretty good, and the Bayesian $D_{s}$-optimal augmenting criterion tends to produce designs with higher Power and Coverage, and lower Type I error rates than the Bayesian $D$-optimal augmenting criterion.

Ruggoo and Vandebroek (2004) pointed out the Bayesian $D$ - $D$ optimal designs coming from two-stage procedures perform more robust and better in comparison to its unique stage competitors, the classical one-stage $D$-optimal and the one-stage Bayesian $D$-optimal designs. For an augmented SSD, since the added design is generated from the Bayesian $D_{s}($ or $D)$ -

optimal procedure that incorporates the improved model knowledge from the first stage and the experts' advice, we may expect such an augmented SSD would be superior in performance to the classical one stage SSDs in some situations and this is also our future work.

There are still some limitations of our simulation studies, more variable selection methods and designs should be considered, it is possible that classification results from much more analysis strategies may be more accurate, and then improve the efficiency of the Bayesian

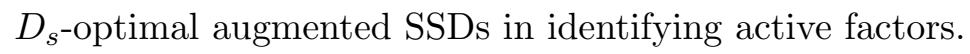

In the literature, most of the SSDs are for main effects only models. However, studies are needed to construct SSDs for the estimation of factor interactions. 


\section{Acknowledgments}

This work was supported by the National Natural Science Foundation of China (Grant Nos. 11771220, 11431006, and 11601244), Tianjin Development Program for Innovation and Entrepreneurship, Tianjin "131" Talents Program, and Project 61331903. The authors thank the Executive-Editor, an Associate-Editor and two referees for their valuable comments.

\section{References}

Abraham, B., Chipman, H. and Vijayan, K. (1999). Some risks in the construction and analysis of supersaturated designs. Technometrics 41, 135-141.

Atkinson, A. C. and Donev, A. N. (1992). Optimum Experimental Designs. Oxford Science Publications, Oxford.

Beattie, S. D., Fong, D. K. H. and Lin, D. K. J. (2002). A two-stage Bayesian model selection strategy for supersaturated designs. Technometrics 44, 55-63.

Booth, K. H. V. and Cox, D. R. (1962). Some systematic supersaturated designs. Technometrics 4, 489-495.

Box, G. E. P. (1959). Discusssion on "Random balance experimentation" by F. E. Satterthwaite. Technometrics 1, 174-180.

Box, G. E. P., Meyer, R. D. (1986). Ananalysis for unreplicated fractional factorials.Technometrics $28,11-18$.

Candes, E. and Tao, T. (2007). The Dantzig selector: statistical estimation when $p$ is much larger than $n \mathrm{n}$ (with discussion). The Annals of Statistics 35, 2313-2351.

Casey, M., Gennings, C., Carter, W. H. J., Moser, V. C. and Simmons, J. E. (2005). $D_{s}$-optimal designs for studying combinations of chemicals using multiple fixed-ratio ray experiments. Environmetrics $16,129-147$.

Chen, R. B., Chu, C. H., Lai, T. Y. and Wu, Y. N. (2011). Stochastic matching pursuit for Bayesian variable selection. Statistics and Computing 21, 247-259.

Chen, R. B., Weng, J. Z. and Chu, C. H. (2013). Screening procedure for supersaturated designs using a Bayesian variable selection method. Quality and Reliability Engineering International 29, 89-101. 
Daniel, C. (1959). Use of half-normal plots in interpreting factorial two-level experiments. Technometrics 1, 311-341.

DuMouchel, W. and Jones, B. (1994). A simple bayesian modification of $D$-optimal designs to reduce dependence on an assumed model. Technometrics 36, 37-47.

Fan, J. (1997). Comments on "Wavelets in statistics: a review" by A. Antoniadis. Journal of the Italian Statistical Society 6, 131-138.

Fan, J. and Li, R. (2001). Variable selection via nonconcave penalized likelihood and its oracle properties. Journal of the American Statistical Association 96, 1348-1360.

Georgiou, S. D. (2014). Supersaturated designs: a review of their construction and analysis. Journal of Statistical Planning and Inference 144, 92-109.

Goos, P. and Jones, B. (2011). Optimal design of experiments: a case study approach. John Wiley and Sons, New York.

Gupta, V. K., Chatterjee, K., Das, A. and Kole, B. (2012). Addition of runs to an s-level supersaturated design. Journal of Statistical Planning and Inference 142, 2402-2408.

Gupta, V. K., Singh, P., Kole, B. and Parsad, R. (2010). Addition of runs to a two-level supersaturated design. Journal of Statistical Planning and Inference 140, 2531-2535.

Gutman, A. J., White, E. D., Lin, D. K. J. and Hill, R. R. (2014). Augmenting supersaturated designs with Bayesian D-optimality. Computational Statistics and Data Analysis 71, 1147-1158.

Huang, H. Z., Yang, J. Y. and Liu, M. Q. (2014). Functionally induced priors for componentwise Gibbs sampler in the analysis of supersaturated designs. Computational Statistics and Data Analysis 72, $1-12$.

Jones, B., Lin, D. K. J. and Nachtsheim, C. J. (2008). Bayesian D-optimal supersaturated designs. Journal of Statistical Planning and Inference 138, 86-92.

Kiefer, J. and Wolfowitz, J. (1961). Optimum designs in regression problems. Annals of Mathematical Statistics 32, 298-325.

Li, R. and Lin, D. K. J. (2002). Data analysis in supersaturated designs. Statistics and Probability Letters 59, 135-144.

Lin, D. K. J. (1993). A new class of supersaturated designs. Technometrics 35, 28-31.

Lin, D. K. J. (1995). Generating systematic supersaturated designs. Technometrics 37, 213-225. 
Marley, C. J. and Woods, D. C. (2010). A comparison of design and model selection methods for supersaturated experiments. Computational Statistics and Data Analysis 54, 3158-3167.

Nguyen, N. (1996). An alogrithmic approach to constructing supersaturated designs. Technometrics $38,69-73$.

Phoa, F. K. H., Pan, Y. H. and Xu, H. (2009). Analysis of supersaturated designs via the Dantzig selector. Journal of Statistical Planning and Inference 139, 2362-2372.

Qin, H., Chatterjee, K. and Ghosh, S. (2015). Extended mixed-level supersaturated designs. Journal of Statistical Planning and Inference 157, 100-107.

Ruggoo, A. and Vandebroek, M. (2004). Bayesian sequential $D$ - $D$ optimal model-robust designs. Computational Statistics and Data Analysis 47, 655-673.

Salawu, I. S., Adeleke, B. L. and Oyeyemi, G. M. (2015). Review of classical methods in supersaturated designs (SSD) for factor screening. Mathematical Theory and Modeling 5, 38-44.

Suen, C. and Das, A. (2010). E( $\left.s^{2}\right)$-optimal supersaturated designs with odd number of runs. Journal of Statistical Planning and Inference 140, 1398-1409.

Sun, F. S., Lin, D. K. J. and Liu, M. Q. (2011). On construction of optimal mixed-level supersaturated designs. Annals of Statistics 39, 1310-1333.

Tibshirani, R. J. (1996). Regression shrinkage and selection via the lasso. Journal of the Royal Statistical Society: Series B (Statistical Methodology) 58, 267-288.

Westfall, P. H., Young, S. S. and Lin, D. K. J. (1998). Forward selection error control in the analysis of supersaturated designs. Statistica Sinica 8, 101-117.

Wu, C. F. J. (1993). Construction of supersaturated designs through partially aliased interactions. Biometrika 80, 661-669.

Yin, Y. H., Zhang, Q. Z. and Liu, M. Q. (2013). A two-stage variable selection strategy for supersaturated designs with multiple responses. Frontiers of Mathematics in China 8, 717-730.

Zhang, Q. Z., Zhang, R. C. and Liu, M. Q. (2007). A method for screening active effects in supersaturated designs. Journal of Statistical Planning and Inference 137, 2068-2079. 